KANSAS JOURNAL of MEDICINE

\section{Return to Sport after Surgical Treatment of Lisfranc Injuries in Athletes: A Retrospective Case Series}

Bryan G. Vopat, M.D. ${ }^{1}$, Matthew L. Vopat, M.D. ${ }^{2}$, Pim A.D. van Dijk, B.Sc. ${ }^{3-6}$, Sean Hazzard, PA-C ${ }^{4}$, Kayla McKinnon, PA-C ${ }^{4}$, Peter D. Asnis, M.D. ${ }^{3}$, George H. Theodore, M.D. ${ }^{3}$

${ }^{1}$ University of Kansas Medical Center, Kansas City, KS

${ }^{2}$ University of Kansas School of Medicine-Wichita, Wichita, KS

${ }^{3}$ Academic Medical Center, University of Amsterdam, the Netherlands

${ }^{4}$ Massachusetts General Hospital/Harvard University, Boston, MA

${ }^{5}$ Academic Center for Evidence-based Sports Medicine, Amsterdam, the Netherlands

${ }^{6}$ Amsterdam Collaboration on Health and Safety in Sports, Amsterdam, the Netherlands

Received April 1, 2019; Accepted for publication.June 26, 2019; Published online Nov. 25, 2019

\begin{abstract}
Introduction. A Lisfranc injury can be a devastating injury in athletes, and if inadequately treated, may lead to chronic pain and loss of function. The purpose of this study was to determine the rate and time until return to sport after surgical fixation for a ligamentous Lisfranc injury. We hypothesized that open reduction and screw fixation of a ligamentous Lisfranc injury can be a successful treatment in the athletic population and allow patients to return to sport at close to their preinjury level of play.
\end{abstract}

Methods. All patients who were analyzed underwent repair of a ligamentous Lisfranc injury with open reduction and screw fixation by a single surgeon, were between 18 - 40 years old at time of their final follow up, and were identified as being an athlete (either recreational or competitive). Eligible patients were given a questionnaire that included if they were able to return to sport, time until return to sport, subjective percentage of pre-injury level of play, current pain (0 - 10), and complications.

Results. Eleven patients were identified as athletes. Ten (91\%) were available for follow-up with a mean of 36.5 months (range, 14 - 60). The average age was 25.4 years (range, 15 - 37) at time of surgery. Eighty percent (8/10) were able to return to sport. The average time until return to sport was 29.4 weeks (range, 22 - 52) with an average subjective value of their pre-injury level of play of $87 \%$ (range, 70 $100 \%)$. However, $67 \%$ (6/9) of the athletes had occasional pain with sport with an average pain level of 2.1 (range, 0 - 5). Two patients had complications, a superficial infection and a deep vein thrombosis.

Conclusion. Most athletes were able to return to sport after undergoing open reduction and internal fixation of a ligamentous Lisfranc injury by less than 30 weeks post-surgery with a subjective value of $87 \%$ of their previous function. However, the majority of the patients also experienced some residual pain with their respective sport. These findings suggested that athletes with a ligamentous Lisfranc injury can have reliably good outcomes with operative repair.

\section{Kans J Med 2019;12(4):141-145. INTRODUCTION}

A Lisfranc injury can be debilitating and, if left untreated, may result in a career ending injury that can continue after their sport career with chronic pain, loss of function, and long term consequences of arthritis. ${ }^{1-4}$ While Lisfranc injuries account for a relatively small percentage of foot injuries with an incidence of approximately 1 in 55,000 persons every year; ${ }^{1}$ these injuries increasingly have been diagnosed in athletes. ${ }^{5}$ Lisfranc injuries have been reported in a broad variety of sports including football, soccer, baseball, basketball, cross country, hockey, gymnastics, and windsurfers, and may occur at all levels of competition. . $^{25-7}$

In athletes, the injury may result from axial load or excessive supination or pronation to the plantar flexed foot. ${ }^{8}$ Injuries to the Lisfranc ligament complex comprise large spectrum from nondisplaced sprains to complete tears with frank diastasis. ${ }^{6}$ In relatively mild injuries, only the dorsal ligament is involved and the plantar ligament remains intact. More severe traumas are associated with tears in both structures, resulting in an unstable Lisfranc joint and a dorsal dislocation of the metatarsal bones. ${ }^{9}$ While severe injuries with obvious deformity are easier to diagnose, low-energy trauma mechanisms often result in subtle symptoms and clinical findings which make accurate diagnosis challenging.

The management strategy of Lisfranc injuries depends on the severity of the disorder. Conservative management is often sufficient in nondisplaced injuries which are stable to stress testing. ${ }^{3,410-12}$ Patients with unstable, displaced injuries, however, require anatomical surgical reduction. Although good clinical outcomes have been reported with different operative procedures, orthopedic literature is limited on operative treatment of ligamentous Lisfranc injuries and the correlation with returning to sporting activities. The majority of the studies that look at athletes combine bony Lisfranc and ligamentous Lisfranc injuries and few look specifically at ligamentous Lisfranc injuries when reporting the return to sport. The purpose of this study was to determine the return to sport time and rate in ligamentous Lisfranc injuries treated with open reduction and operative fixation. We hypothesized that open reduction and screw fixation of a ligamentous Lisfranc injury can be a successful treatment in the athletic population and allow patients to return to sport at close to their preinjury level of play.

\section{METHODS}

The Institutional Review Board at the hospital where the senior author practices approved this retrospective study.

Subjects. Patients who underwent open reduction and internal fixation (ORIF) for ligamentous Lisfranc injuries by the senior author between the years of 2010 to 2014 were identified using billing records and operative reports. Decision for operative management was based on the classification of Nunley et al., ${ }^{6}$ when displacement 
was greater than $2 \mathrm{~mm}$, between the first and second metatarsals on weight bearing anteroposterior foot radiograph. A single surgeon performed all surgical procedures and provided both pre- and postoperative care.

Patients were included if they: (1) were involved in competitive athletics (either recreational or competitive) at their initial visit, (2) were between 18 - 40 years of age at follow-up, and (3) had a minimum follow-up of one year after surgery. When patients underwent a subsequent surgery that confounded meaningful postoperative outcome analysis, they were excluded from the study.

Data Collection. Radiographic images (weight bearing anteroposterior, oblique, and lateral x-rays) and demographic information were reviewed for each patient. All patients had a primarily ligamentous injury, therefore, the injuries were graded using the Nunley classification. ${ }^{6}$ Details of each surgical procedure were recorded, including type of surgery and complications. All patients were interviewed at final follow-up and a questionnaire was completed with regard to their clinical course. The questionnaire included type of sport they perform, if they were able to return to sport, time until return to sport, subjective percentage of pre-injury level of play, visual analog scale pain score on a scale from 0 to 10 , and complications.

Treatment. Operative treatment was performed by open reduction and internal screw fixation under regional block. A longitudinal dorsal incision was created, and the space between the medial cuneiform and base of the second metatarsal was debrided. Before reduction was performed, the bone surfaces of the medial corner of the base of the second metatarsal and the lateral corner of the distal medial cuneiform were roughened with a burr to create a bony union. Fixation consisted of a single fully threaded $4.5 \mathrm{~mm}$ screw between the medial cuneiform and second metatarsal (Figure 1). If patients had intercuneiform instability after this screw was placed, a second $4.5 \mathrm{~mm}$ intercuneiform screw was inserted from the medial cuneiform into the middle cuneiform.
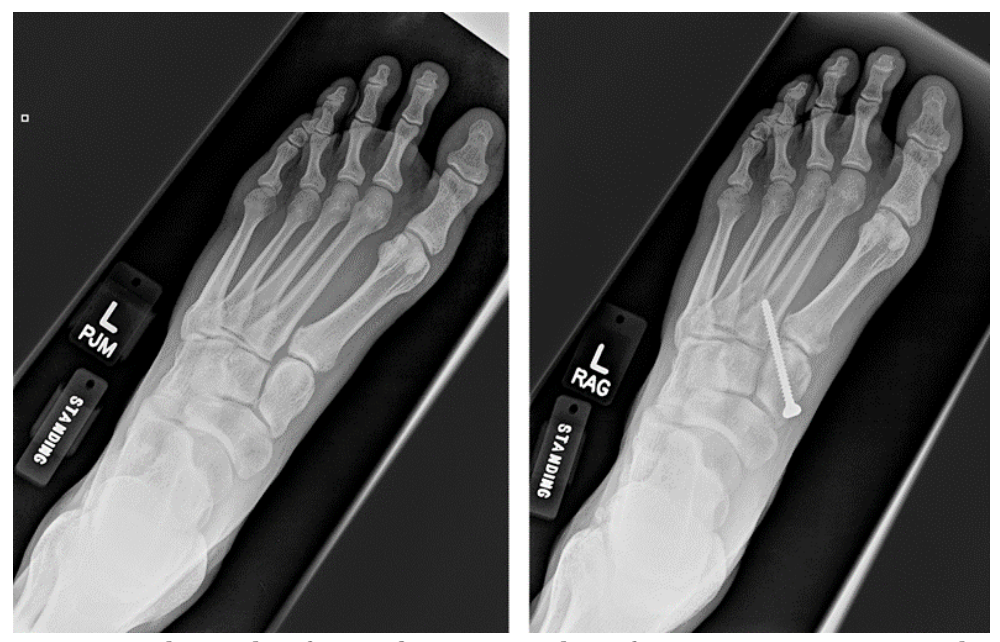

Figure 1. Radiographs of screw fixation sample. Left: an anteroposterior standing radiograph demonstrating diastasis between the medial cuneiform and $2^{\text {nd }}$ metarsal. Right: status post open reduction and fixation with single screw between the medial cuneifrom and $2^{\text {nd }}$ metatarsal.
KANSAS JOURNAL of MEDICINE LISFRANC INJURIES IN ATHLETES

continued.

Following surgical treatment patients were splinted for two weeks, non-weight-bearing. Sutures were removed at two weeks and the patient was placed in a bivalved cast. They were instructed to remain non-weight-bearing, but were encouraged to remove the cast daily for ankle and toe range of motion exercises. At six weeks post-surgery, a standing $x$-ray was reviewed to check maintained alignment. Patients were placed in a walking boot and began weight-bearing as tolerated. Twelve weeks postoperatively, a second x-ray further assessed alignment and healing. At this point, hardware removal was discussed with the patient and routinely was removed. Following the hardware removal, patients were placed in a boot and weaned out as tolerated. Formal physical therapy at this point focused on proprioception and progressed to strengthening exercises. Through a dedicated physical therapy course, patients were able to return to sport when they had regained almost full ankle plantar flexion strength and perform a single limb heel raise without pain.

\section{RESULTS}

During the study period, 11 patients who had a ligamentous Lisfranc injury treated with surgical repair were identified as athletes, of which $91 \%$ (10/11) were available for at least one year of followup with an average follow up of 36.5 (range, 14 - 60) months. The one patient that was not available for follow-up was excluded from all analysis. The mean age at time of surgery was 25.4 (range, 15 37 ) years. Only one patient had a final first metatarsal to medial cuneiform diastasis of greater than $2 \mathrm{~mm}(3 \mathrm{~mm})$. Further patient demographics are shown in Table 1.

All patients returned to training (100\%) after a mean time of 27.1 (range, 16 - 56) weeks. Eight patients returned to full competition $(80 \%)$. One patient did not attempt to return to play because of a chronic knee injury that was treated by another physician. Another patient had a four-month delay in treatment and attempted to return to sport, but was unable to achieve full competition. Time until return to full competition was 29.4 (range, 22 - 52) weeks. The mean subjective value of their pre-injury level of play was $87 \%$ (range, $70 \%$ - 100\%). Sixty-seven percent (6/9) of the athletes still experienced occasional pain during sporting activities with a mean pain level of 2.1 ( 0 - 5). One patient was excluded from the analysis of pain scale with play because he did not attempt to return to sport due to a chronic knee injury. Complete results can be seen in Table 2.

Complications were reported in two patients, including a superficial infection treated successfully with oral antibiotics and a deep vein thrombosis successfully treated with oral warfarin for six months. 


\section{KANSAS JOURNAL of MEDICINE}

LISFRANC INJURIES IN ATHLETES

continued.

Table 1. Patient demographics.

\begin{tabular}{|c|c|c|c|c|c|c|c|}
\hline Patient & Sport & $\begin{array}{l}\text { Competitive or Recreational } \\
\text { (Level) }\end{array}$ & $\begin{array}{l}\text { Nunley } \\
\text { Classification }\end{array}$ & $\begin{array}{l}\text { Age } \\
\text { (years) }\end{array}$ & $\begin{array}{l}\text { Follow-up } \\
\text { (months) }\end{array}$ & $\begin{array}{l}\text { Initial Diastasis* } \\
(\mathrm{mm})\end{array}$ & $\begin{array}{l}\text { Final Diastasis* } \\
(\mathrm{mm})\end{array}$ \\
\hline 1 & Soccer & Competitive (High School) & 2 & 16 & 48 & 3 & 1 \\
\hline 2 & Soccer & Recreational & 2 & 32 & 18.2 & 4 & $\mathbf{0}$ \\
\hline 3 & Football & Competitive (High School) & 2 & 15 & 60 & 3 & 3 \\
\hline 4 & Cross Training & Recreational & 2 & 36 & 60 & 3 & $\mathbf{0}$ \\
\hline 5 & Softball & Recreational & 3 & 26 & 14 & 7 & $\mathbf{0}$ \\
\hline 6 & Baseball & Competitive (Professional) & 2 & 28 & 24 & 4 & $\mathbf{0}$ \\
\hline 7 & Cross Training & Recreational & 2 & 27 & 24 & 2 & 1 \\
\hline 8 & Wrestling/Football & Competitive (High School) & 3 & 17 & 42 & 6 & 2 \\
\hline 9 & Baseball & Recreational & $\mathbf{N A}$ & 37 & 60 & NA & 1 \\
\hline 10 & Football & Competitive (College) & 2 & 20 & 15 & 4 & 2 \\
\hline \multicolumn{4}{|c|}{ Average \pm SD } & $\begin{array}{l}25.4 \\
\pm 8.1\end{array}$ & $36.5 \pm 20$ & 4 & 1 \\
\hline
\end{tabular}

*First metatarsal to medial cuneiform diastasis.

Table 2. Post-operative results.

\begin{tabular}{|c|c|c|c|c|c|c|c|c|}
\hline Patient & $\begin{array}{l}\text { Removal of } \\
\text { Hardware } \\
\text { Timing } \\
\text { (weeks) }\end{array}$ & $\begin{array}{l}\text { Return to } \\
\text { Training } \\
\text { (weeks) }\end{array}$ & $\begin{array}{l}\text { Full } \\
\text { Competition } \\
\text { (weeks) }\end{array}$ & $\begin{array}{l}\text { Time to } \\
\text { Pre-injury } \\
\text { Status } \\
\text { (weeks) }\end{array}$ & $\begin{array}{l}\text { Pain Scale } \\
\text { with Sport }\end{array}$ & $\begin{array}{l}\text { Percent of } \\
\text { Normal }\end{array}$ & Type of Fixation & $\begin{array}{l}\text { Complications/ } \\
\text { Notes: }\end{array}$ \\
\hline 1 & 13 & 56 & No & No & NA & 95 & $\mathrm{MT}+\mathrm{IC}$ & $\begin{array}{l}\text { Gave up sports } \\
\text { due to other } \\
\text { injuries }\end{array}$ \\
\hline 2 & 16 & 16 & No & No & 3 & 80 & MT & $\begin{array}{c}\text { Delayed ORIF by } \\
4 \text { months }\end{array}$ \\
\hline 3 & 13 & 22 & 22 & 17 & 4 & 75 & MT & $\begin{array}{l}\text { Broken screw } \\
\text { removed }\end{array}$ \\
\hline 4 & 17 & 39 & 52 & 52 & 2 & 75 & $\mathrm{MT}+\mathrm{IC}$ & $\begin{array}{l}\text { ORIF base of } 4 \text { th } \\
\text { MT, Superficial } \\
\text { Infection }\end{array}$ \\
\hline 5 & 15 & 26 & 26 & 26 & 5 & 70 & MT & \\
\hline 6 & 13 & 17 & 26 & 43 & 0 & 100 & $\mathrm{MT}+\mathrm{IC}$ & \\
\hline 7 & 15 & 30 & 35 & 26 & 3 & 85 & $\mathrm{MT}+\mathrm{IC}$ & $\begin{array}{l}\text { ORIF navicular, } \\
\text { Patella tendon } \\
\text { tear slowed RTS } \\
\text { per patient }\end{array}$ \\
\hline 8 & 13 & 17 & 22 & 22 & 2 & 95 & MT & \\
\hline 9 & 13 & 26 & 30 & 22 & 0 & 93 & MT & \\
\hline 10 & 17 & 22 & 22 & 22 & 0 & 100 & $\mathrm{MT}+\mathrm{IC}$ & $\begin{array}{l}\text { DVT treated with } \\
\text { coumadin }\end{array}$ \\
\hline Average \pm SD & $14.5 \pm 1.7$ & $27.1 \pm 12.3$ & $29.4 \pm 10.2$ & $28.8 \pm 12.2$ & $2.1 \pm 1.8$ & $87 \% \pm 11 \%$ & & \\
\hline
\end{tabular}

MT = screw from medial cuneiform to $2^{\text {nd }}$ metatarsal; IC = Intercuneiform screw; ORIF = open reduction and internal fixation; RTS = return to sports 


\section{DISCUSSION}

Our study demonstrated that people reliably can return to sport at an average of 29.4 weeks after undergoing operative fixation for a ligamentous Lisfranc injury. The majority of the athletes were able to return to sport at close to their original subjective level of play. The reason the two athletes were not able to return to competition was because one had other injuries (patella chondromalacia) and the other had a delay in treatment for four months. The majority of patients continued to have some pain with their respective sport. Current literature lacks substantial information for injured athletes with ligamentous Lisfranc injuries with respect to ability to return to sport as well as the timeline for return to their respective sport after surgery. ${ }^{6,13-15}$ This analysis provided a baseline for healthcare providers as well as patients to have an educated discussion on estimated return to play after a ligamentous Lisfranc injury.

Few papers have looked at only ligamentous injuries without including bony Lisfranc injuries. However, Nunley et al. ${ }^{6}$ described 15 athletes with a ligamentous Lisfranc injury. Eight patients underwent operative treatment and were able to return to sport within an average of 14.4 weeks. A recent study by Deol et al. ${ }^{13}$ divided Lisfranc injuries into ligamentous and bony injuries in professional rugby and soccer athletes. They reported on 17 patients, but only seven had pure ligamentous only injuries. One athlete retired after a ligamentous injury; the remaining six players returned to competing at 22.5 weeks, which was significantly less than bony injuries at 26.9 weeks $(p=0.003)$, indicating there may be a difference between the two injuries.

Our athletes returned to competition later (at 29.4 weeks), but we had fewer professional athletes who may be compelled to return sooner to play than recreational and nonprofessional athletes. Other studies have demonstrated the adverse effects that this injury can have on an athlete's career. Chilvers et al. ${ }^{14}$ described surgical treatment of a Lisfranc fracture dislocation injury in five female gymnasts, of which only one was able to return to full competition. The other athletes, retired from gymnastics, were lost to follow-up, or graduated from college. The authors concluded that "Lisfranc injuries were most likely career-ending." ${ }^{14}$ More recently, McHale et al. ${ }^{15}$ looked at the return to play outcomes in 28 national football players with Lisfranc injuries. They found that with patients that needed surgical intervention for their Lisfranc injury that the average return to sport was 49.9 (range; 46.0 - 54.2) weeks. In patients that were treated nonoperatively, their average return to sport was 26.7 (range; 8.2 - 46.01) weeks. Unlike the present study, the McHale study did not distinguish between pure-ligamentous and/or fracture dislocation injuries. Also, they did not differentiate the type of fixation these athletes underwent and their patient population was strictly professional athletes compared to our study which was more representative of general athlete population.

Many authors have advocated percutaneous reduction and fixation for low energy Lisfranc injuries. They argued that when open fixation is performed, greater soft tissue damage is likely to occur. ${ }^{16-19}$ Bleazey et al. ${ }^{18}$ reported anatomical alignment in all 13 patients that were treated with percutaneous screw fixation of bony Lisfranc fractures in athletes and reported a mean return to sports of 16.6 weeks.
KANSAS JOURNAL of MEDICINE LISFRANC INJURIES IN ATHLETES

continued.

An accelerated weight-bearing protocol and return to sport using percutaneous reduction and screw fixation for low-energy Lisfranc fracture dislocation injuries also was advocated by Wagner et al. ${ }^{19}$ who reported a return to training for low-impact sports in all patients at a mean time of 7.6 weeks with symptom free sport activities at a mean of 12.4 weeks. Certain Lisfranc injuries may be more amenable to percutaneous reduction and fixation, but long-term prospective studies are needed to decide whether percutaneous or the open technique is the better option. The senior author believes by doing an open anatomic reduction, and stimulating the opposing bone surfaces between the medial cuneiform and second metatarsal, the patient is likely to form a bone bridge which increases the stability of the injured area long-term and prevents late loss of reduction.

Other studies have recommended primary fusion for ligamentous Lisfranc injuries for patients to return to their previous physical activities. ${ }^{20,21}$ Macmahon et al. ${ }^{20}$ investigated 38 patients with only six of them being purely ligamentous injuries that underwent primary partial arthrodesis for Lisfranc injury. Nevertheless, they found no difference in sport activity and clinical outcomes between combined and purely ligamentous Lisfranc injuries. They also found that most of their patients returned to their previous physical activities post-operatively with the majority being high impact activities. Nevertheless, they concluded that with the decreased participation or increased difficulty of some activities found in their results, some patients experienced postoperative limitations in exercise. ${ }^{20}$ Ly et al. ${ }^{21}$ compared the difference in outcomes between primary arthrodesis with open reduction and internal fixation of 41 primarily ligamentous Lisfranc injuries. Their results revealed with the primary arthrodesis population that they returned to $92 \%$ of their pre-injury level with physical and sport activities and only $65 \%$ in the open reduction and internal fixation population. Furthermore, due to the poor healing potential of the ligament-osseous interface and a high rate of correction loss, increasing deformity, and degenerative arthritis changes, they believed that primary arthrodesis was superior in short and mediumterm outcomes. Though contrast to the current study which looked at an athletic population, their study represented the general population.

Although multiple approaches to the repair of Lisfranc injuries have been advocated most authors agree that anatomic reduction is the most important goal to regain future function., ${ }^{40}$ According to Hardcastle et al. ${ }^{22}$, the prognosis of a Lisfranc injury depends on achieving and maintaining accurate reduction while preserving the posture of the midfoot. Since Lisfranc injuries cover a broad spectrum of injuries, Coetzee et al. ${ }^{9}$ suggested that no single treatment option will be defined for all injuries. In the current study, treatment was adjusted to each individual patient, all with the primary intention to obtain accurate anatomic reduction.

Our study is not without limitations. Results are based on a relatively small number of patients and data were collected retrospectively. However, there was only one patient lost to follow-up. 
KANSAS JOURNAL of MEDICINE LISFRANC INJURIES IN ATHLETES continued.

Moreover, since return to sports is monitored accurately during regular outpatient appointments in our institution, recall bias was not taken into account. Also, the senior author has a slight deviation in his operative technique, using a burr to form a bony union between the medial corner of the base of the second metatarsal and the lateral corner of the distal medial cuneiform. This particular step is unique to our study and a potential variable that could have resulted in the difference in our outcomes to what previous literature reported. Furthermore, our study population did not allow us to compare the different levels of competition. Also, our study did not follow these patients for several years to assess for the development of arthritis or the functional long-term outcome of a bone bridge.

\section{CONCLUSIONS}

Most athletes were able to return to sport after undergoing open reduction and internal fixation of a ligamentous Lisfranc injury by less than 30 weeks post-surgery with a subjective value of $87 \%$ of their previous function. However, the majority of the patients also experienced some residual pain with their respective sport. These findings suggested that athletes with a ligamentous Lisfranc injury can have reliably good outcomes with operative repair.

\section{REFERENCES}

${ }^{1}$ Desmond EA, Chou LB. Current concepts review: Lisfranc injuries. Foot Ankle Int 2006; 27(8):653-660. PMID: 16919225.

2 Eleftheriou KI, Rosenfeld PF. Lisfranc injury in the athlete: Evidence supporting management from sprain to fracture dislocation. Foot Ankle Int 2013; 18(2):219-236. PMID: 23707175.

3 Stavlas P, Roberts CS, Xypnitos FN, Giannoudis PV. The role of reduction and internal fixation of Lisfranc fracture-dislocations: A systematic review of the literature. Int Ortho 2010; 34(8):1083-1091. PMID: 2083593.

4 Teng AL, Pinzur MS, Lomasney L, Mahoney L, Havey R. Functional outcome following anatomic restoration of tarsal-metatarsal fracture dislocation. Foot Ankle Int 2002; 23(10):922-926. PMID: 12398144.

${ }^{5}$ Meyer SA, Callaghan JJ, Albright JP, Crowley ET, Powell JW. Midfoot sprains in collegiate football players. Am J Sports Med 1994; 22(3):392-401. PMID: 7913587.

${ }^{6}$ Nunley JA, Vertullo CJ. Classification, investigation, and management of midfoot sprains: Lisfranc injuries in the athlete. Am J Sports Med 2002; 30(6):871-878. PMID: 12435655.

7 Miyamoto W, Takao M, Innami K, Miki S, Matsushita T. Ligament reconstruction with single bone tunnel technique for chronic symptomatic subtle injury of the Lisfranc joint in athletes. Arch Orthop Trauma Surg 2015; 135(8):1063-1070. PMID: 26018890.

${ }^{8}$ Kent RW, Lievers WB, Riley PO, Frimenko RE, Crandall JR. Etiology and biomechanics of tarsometatarsal injuries in professional football players: A video analysis. Orthop J Sports Med 2014; 2(3):2325967114525347. PMID: 26535308.

9 Coetzee JC. Making sense of lisfranc injuries. Foot Ankle Clin 2008; 13(4):695-704, ix. PMID: 19013403.

10 Kuo RS, Tejwani NC, Digiovanni CW, et al. Outcome after open reduction and internal fixation of Lisfranc joint injuries. J Bone Joint Surg Am 2000; 82-A(11):1609-1618. PMID: 11097452.

${ }^{11}$ Osbahr DC, O'Loughlin PF, Drakos MC, Barnes RP, Kennedy JG, Warren RF. Midfoot sprains in the National Football League. Am J Orthop (Belle Mead NJ) 2014; 43(12):557-561. PMID: 25490010.

${ }^{12}$ Lorenz DS, Beauchamp C. Functional progression and return to sport criteria for a high school football player following surgery for a lisfranc injury. Int J Sports Phys Ther 2013; 8(2):162-171. PMID: 23593554.
13 Deol RS, Roche A, Calder JD. Return to training and playing after acute Lisfranc injuries in elite professional soccer and rugby players. Am J Sports Med 2016; 44(1):166-170. PMID: 26637283.

${ }^{14}$ Chilvers M, Donahue M, Nassar L, Manoli A 2nd. Foot and ankle injuries in elite female gymnasts. Foot Ankle Int 2007; 28(2):214-218. PMID: 17296142.

${ }_{15}$ McHale KJ, Rozell JC, Milby AH, Carey JL, Sennett BJ. Outcomes of Lisfranc injuries in the National Football League. Am J Sports Med 2016; 44(7):1810-1817. PMID: 27166291.

${ }^{16}$ Perugia D, Basile A, Battaglia A, Stopponi M, De Simeonibus AU. Fracture dislocations of Lisfranc's joint treated with closed reduction and percutaneous fixation. Int Orthop 2003; 27(1):30-35. PMID: 12582806.

${ }^{17}$ Richter M, Wippermann B, Krettek C, Schratt HE, Hufner T, Therman H. Fractures and fracture dislocations of the midfoot: Occurrence, causes and long-term results. Foot Ankle Int 2001; 22(5):392-398. PMID: 11428757.

18 Bleazey ST, Brigido SA, Protzman NM. Technique tip: Percutaneous fixation of partial incongruous Lisfranc injuries in athletes. Foot Ankle Spec 2013; 6(3):217-221. PMID: 23631892.

19 Wagner E, Ortiz C, Villalón IE, Keller A, Wagner P. Early weight-bearing after percutaneous reduction and screw fixation for low-energy lisfranc injury. Foot Ankle Int 2013; 34(7):978-983. PMID: 23386753.

20 MacMahon A, Kim P, Levine DS, et al. Return to sports and physical activities after primary partial arthrodesis for Lisfranc injuries in young patients. Foot Ankle Int 2016; 37(4):355-362. PMID: 26596795.

${ }^{21}$ Ly TV, Coetzee JC. Treatment of primarily ligamentous Lisfranc joint injuries: Primary arthrodesis compared with open reduction and internal fixation. A prospective, randomized study. J Bone Joint Surg Am 2006; 88(3):514-520. PMID: 16510816.

${ }^{22}$ Hardcastle PH, Reschauer R, Kutscha-Lissberg E, Schoffmann W. Injuries to the tarsometatarsal joint. Incidence, classification and treatment. J Bone Joint Surg Br 1982; 64(3):349-356. PMID: 7096403.

Keywords: orthopedics, operative surgical procedures, injury, sports, athletes 